\title{
Retrospective Analysis of Prevalence of High-risk and Low-risk Human Papillomavirus (HPV) Genotypes in Iranian Women During 2013-2016
}

\author{
Siavash Chalabiani ${ }^{1}$, Mina Khodadad Nazari ${ }^{1}$, Mahdi Shabani ${ }^{2}$, Neda Razavi \\ Davoodi ${ }^{1}$, Abdolfattah Sarafnejad ${ }^{1,3}$, Ali Akbar Amirzargar ${ }^{1,4,5}$
}

${ }^{1}$ Department of Molecular Diagnostics, NOOR Pathobiology Laboratory, Tehran, Iran. ${ }^{2}$ Department of Immunology, School of Medicine, Shahid Beheshti University of Medical Sciences, Tehran, Iran. ${ }^{3}$ Department of Immunology, School of Public Health, Tehran University of Medical Sciences, Tehran, Iran. ${ }^{4}$ Department of Immunology, School of Medicine, Tehran University of Medical Sciences, Tehran, Iran. ${ }^{5}$ Molecular Immunology Research Center, Tehran University of Medical Sciences, Tehran, Iran.

\begin{abstract}
Background and objective: Considering the importance of HPV in cancer etiology, awareness of HPV prevalence and frequency of high-risk genotypes could help improve health care system management. We analyzed HPV prevalence in women forms different provinces of Iran that is the largest sample till now. Material and Methods: In this retrospective cross-sectional study, 2969 outpatient and suspicious women aged 17- 78 referred to Noor pathobiology laboratory from 24 provinces of Iran were studies. DNA extraction and PCR were performed on samples and then HPV genotypes were also determined using hybridization kit. Results: HPV DNA was detected in $29.3 \%$ of valid liquid-based samples. Among HPV positive cases, $67.2 \%$ and $52.0 \%$ accounted for high-risk and low-risk HPV subtypes, respectively. In patients with high-risk HPV types, HPV16 was confirmed as predominate type (30.5\%) followed by HPV53 (17.3\%) and HPV39 (13.3\%). HPV6 was found as the most common low-risk HPV type with $60.6 \%$ frequency rate followed by HPV11 $(17.9 \%)$ and HPV81 $(8.6 \%)$ in this age group. It was apparent that age group $>25$ years accounted the highest frequency of HPV positivity. The prevalence of HPV was significantly different among different age groups $(p<0.0001)$. Conclusion: It can be concluded that HPV infection is currently at a considerable high level in Iran. Looking at the high risk and oncogenic HPV subtypes frequency especially in younger age groups, a concern could be noticed about HPV relevant cancers which can be prevented by commercial and approved HPV vaccines.
\end{abstract}

Keywords: Genotypes- high risk- human papillomavirus- low risk- prevalence

Asian Pac J Cancer Biol, 2 (4), 85-90

\section{Introduction}

Human papillomaviruses (HPVs) family consists of more than 200 different types of non enveloped and doublestranded DNA viruses that can exclusively infect stratified squamous cutaneous cells or the mucosa of upper-respiratory and genitals tracts [1-2]. These viruses have worldwide dispersal that can be recognized based on their oncogenic potential, biological niche, and phylogenetic state. In the genital tract, about $40 \mathrm{HPV}$ genotypes are generally found $[3,4]$. HPV is one the most
Submission Date: 09/12/2017 Acceptance Date: 11/25/2017

common sexually transmitted agent that affects young and sexually active people of equal sexes [5-6]. Genital HPV types have been subdivided into high risk and low-risk types on the basis of their potential to cause malignant progression [7]. HPV 16, 18, 31, 33, 35, 39, 45, 51, 52, $53,56,58,59,66$, and 68 have been involved in cervical carcinogenesis and categorized as high risk types, while other types, such as HPV 6, 11, 40, 42, 43, 44, 54, 61, 70, 72, 81, and HPV, 89/Cp 6108 are classified as low risk and related to genital warts [8-9].

The most predominant types of high risk HPV

Corresponding Author:

Dr. Ali Akbar Amirzargar

Noor Pathobiology Laboratory \& Department of Immunology, School of Medicine, Tehran University of Medical Sciences.

Email: amirzara@sina.tums.ac.ir 
genotypes in the worldwide are HPV-16 and HPV 18 [10]. Genital wart is the most common benign tumor that is naturally presented on the outside of genitalia, including the penis, scrotum, vulva, perineum, and perianal skin [9, $11,12]$. Spreading of HPV viruses occur through skin or surface contact and the spreading can be horizontal (by genital-genital, manual-genital, or oral-genital contact) or vertical (from mother to fetus) [13, 14].

Cytological and molecular methods have been currently applied for detection and screening of HPV viral infection. Pap smear, as a cytological test, has been generally used as the principal approach for cervical lesion screening; however, it presented considerable false negative results [15-17]. Therefore, molecular assays such as PCR hybridizations, PCR, and Real -time PCR have been introduced for easier and more accurate HPVs detection and genotyping [9].

Prevalence of HPV in developing countries (42.4\%) is more public in comparison to the developed countries (22.6\%) [18]. Out of 233.9 million women at reproductive age worldwide, nearly 32.03 million and 3.71 million were Asian and Iranian women, respectively [19]. In addition, South Africa (58.1\%), Singapore (25.6\%), France (15\%), Russia (13\%), China (12.27\%), Chile (11.1\%), Brazil $(10 \%)$, and Iran $(9.4 \%)$ have been recognized as HPV high prevalence areas based on the epidemiological studies [20-27]. Based on WHO report in 2010, the number of Iranian women aged 15 years and older is more than 25 million who are at risk of cervical carcinoma. The incidence rate of cervical cancer with an age normalization is 2.2 per 100,000 women each year, that is considered as the second most public threat of female reproductive system in Iran [28].

Thus, due to the importance of HPV prevalence and especially high risk genotypes, we persuaded to determine the prevalence of HPV infection and the related high-risk and low-risk genotypes in women from different provinces of Iran. Our findings can be are very informative for decision making on HPV screening and prevention programs by prophylactic vaccines. For achieving this aim, we calculated HPV prevalence rates in a large number of patients referred to Noor pathobiology laboratory.

\section{Materials and Methods}

\section{Patients and sample collection}

In this retrospective cross-sectional study, 2969 outpatient and suspicious women aged 17-78 years old (mean \pm SD $33.4 \pm 8.4$ ) were studied who were referred from 24 out of 31 provinces of Iran to Noor pathobiology laboratory during November 2013 to September 2016. Patients were subdivided into $<25$ years, 25-34 years, $35-45$ years, and $>46$ years age groups accounted for $11.5 \%, 51.9 \%, 26.4 \%$, and $10.2 \%$ of cases, respectively. Brush vaginal swaps $(n=2913)$ and genital warts $(n=56)$ were checked for HPV infection according to prescribed order. Samples were collected by pastoCYTOprep (Pasture Institute of Iran, Tehran, Iran) transport medium.

\section{HPV DNA extraction}

Viral DNA extraction from different samples was carried out by High pure PCR Template preparation kit according to manufacturer's instruction (Roche, Germany). Briefly, genital warts were initially digested using tissue lysis buffer and proteinase $\mathrm{K}$ (Roche, Germany) for $72 \mathrm{hr}$ at $37{ }^{\circ} \mathrm{C}$. Two hundred microliters of each sample was mixed with equal volume of binding buffer and $40 \mu \mathrm{l}$ proteinase $\mathrm{K}$ and incubated for $10 \mathrm{~min}$ at $70{ }^{\circ} \mathrm{C}$. Then, $100 \mu \mathrm{l}$ isopropanol was added and the mixture was applied to a highly pure filter tube and centrifuged for $1 \mathrm{~min}$ at $8000 \times \mathrm{g}$. The filter was washed with $500 \mu$ inhibitor removal buffer and consequently by wash buffer using centrifugation. Flow -through was discarded and DNA was eluted with $200 \mu$ l elution buffer using centrifugation for $1 \mathrm{~min}$ at $8000 \times \mathrm{g}$.

\section{PCR amplification}

HPV DNA amplification was performed by PCR technique using the approved commercial kit (Hybribio 21 HPV GenoArray diagnostic kit, Hybribio, China). For each reaction, $24 \mu \mathrm{l}$ of PCR ready to use master mix, $1 \mu \mathrm{l}$ of DNA Taq polymerase, and $1 \mu$ of extracted DNA were transferred into appropriately labeled tubes. Hot start DNA polymerase was activated by holding at $95^{\circ} \mathrm{C}$ for $9 \mathrm{~min}$ and sequentially cycled for 40 times at $95^{\circ} \mathrm{C}$ for $20 \mathrm{sec}$, $55^{\circ} \mathrm{C}$ for $30 \mathrm{sec}$ and $72{ }^{\circ} \mathrm{C}$ for $30 \mathrm{sec}$ and followed by final extension at $72^{\circ} \mathrm{C}$ for $5 \mathrm{~min}$. Non-infectious plasmid DNA supplemented in the master mix was applied as an internal control in all reactions. Positive and negative controls were used appropriately according to the kit procedure.

\section{Hybridization}

HPV genotypes of amplified DNAs were detected using Hybribio 21 HPV GenoArray diagnostic kit (Hybribio, China). The GenoArrayt kit detected $21 \mathrm{HPV}$ genotypes, including 13 high risk types (HPV 16, 18, $31,33,35,39,45,51,52,56,58,59$, and 68), 6 low risk types (HPV 6, 11, 42, 43, 44, and CP8304 (81). The tests were executed according to the manufacturer's instructions. Briefly, all amplified DNA samples were denatured at $95{ }^{\circ} \mathrm{C}$ for $5 \mathrm{~min}$ and were promptly put into an ice block. Hybridization process was carried out in DNA HybribioMax (Guang gong Kaipu Biotech Co, China) device in 3 different temperature conditions. At first step, HybribioMax was set at $45{ }^{\circ} \mathrm{C}$ and then 0.8 $\mathrm{ml}$ of pre-warmed hybridization solution was added on hybridization probed membrane (HybriMem) located in the well and incubated for $3 \mathrm{~min}$. Then, the buffer was replaced with $0.5 \mathrm{ml}$ fresh hybridization solution mixed with denatured DNA. HybriMem and DNA were incubated for $20 \mathrm{~min}$ at $45^{\circ} \mathrm{C}$. At the second step, HybribioMax was set at $25{ }^{\circ} \mathrm{C}$ and $0.5 \mathrm{ml}$ of blocking buffer was added into the well when the temperature reached around 30 ${ }^{\circ} \mathrm{C}$. Then, $0.5 \mathrm{ml}$ of enzyme conjugate was added and incubated for $5 \mathrm{~min}$. Next, thewell was washed 3 times with wash buffer A. In the last step, HybribioMax was set at $36^{\circ} \mathrm{C}$ and NBT/BCIP solution was added and incubated for $5 \mathrm{~min}$. Finally, the well was washed 3 times with wash 
buffer B and distilled water. The results were interpreted by color visualization observed on the membrane using the provided guide. The average detection limit of the kit, as reported by the manufacturer, was around 300 copies per $\mu$ l of target HPV DNA. No cross-reactivity was shown among all the 21 studied HPV genotypes.

\section{Statistical analysis}

Data were analyzed by SPSS 16 (Chicago, IL, USA). The difference in HPV prevalence in different age groups was compared by Chi-square test. Statistical significance was defined as $\mathrm{p}<0.05$.

\section{Results}

Our results indicated that HPV DNA was detected in

$29.3 \%$ (871 out of 2969$)$ of valid liquid-based samples of which 848/2913 (29.1\%) and 23/56 (41.1\%) were HPV positive in brush vaginal swaps and genital warts, respectively. HPV prevalence was significantly higher in genital warts compared to brush vaginal swaps $(\mathrm{p}=0.05)$. Among HPV positive cases, $67.2 \%$ and $52.0 \%$ accounted for high-risk and low-risk HPV subtypes, respectively (Table 1). Following further data analysis, it was found that
HPV16, HPV18, and other HPV genotypes were in 20.5\%, $7.0 \%$, and $88.4 \%$ of HPV positive samples, respectively. In addition, 563/871 (64.6\%) and 308/871 (35.4\%) of infected subjects were single and multiple HPV types, respectively. In patients with high-risk HPV types, HPV16 was confirmed as the predominate type with $179 / 585$ (30.5\%) rate followed by HPV53 (17.3\%) and HPV39 $(13.3 \%)$ in this category (Table 2). On the other hand, HPV6 was found as the most common low-risk HPV type with $60.6 \%$ frequency rate followed by HPV11 (17.9\%) and HPV81 (8.6\%) in this group (Table 2). Interestingly, HPV6 was the most detected genotype in wart samples (28.6\%) (Table 2).

As presented in Figure 1, HPV was detected in 133/341 (39.0\%), 476/1542 (30.8\%), 195/784 (24.8\%) and 67/302 $(22.1 \%)$ of age groups $<25,25-34,35-45$, and $>45$ years, respectively. It was apparent that age group $>25$ accounted the highest frequency of HPV positivity. In addition, HPV infection decreased from 39.0\% among women aged $<25$ years to $22.1 \%$ in women aged $>45$ years. The prevalence of HPV in the age groups was significantly different $(p<0.0001)$. We did not access to Pap smear results to compare HPV prevalence in cytological normal

Table 1. Prevalence of High-Risk and Low-Risk HPV Types in Women from 21 Different Provinces of Iran

\begin{tabular}{lccc}
\hline HPV & High-risk (\%) & Low-risk (\%) & Total (\%) \\
\hline Positive & $584(19.7)$ & $453(15.3)$ & $871(29.3)$ \\
Negative & - & - & $2098(70.7)$ \\
\hline
\end{tabular}

Table 2. Frequency of HPV Genotypes in 2969 Iranian Women Attended to Noor Pathobiology Lab

\begin{tabular}{|c|c|c|c|c|}
\hline Category & HPV type & Frequency $(\%)$ & Brush swap samples & Genital warts \\
\hline \multirow{15}{*}{ High-risk } & HPV16 & $179(6.0)$ & 177 & 2 \\
\hline & HPV18 & $61(2.1)$ & 59 & 2 \\
\hline & HPV31 & $72(2.4)$ & 72 & 0 \\
\hline & HPV33 & $4(0.1)$ & 4 & 0 \\
\hline & HPV35 & $21(0.7)$ & 21 & 0 \\
\hline & HPV39 & $78(2.6)$ & 78 & 0 \\
\hline & HPV45 & $32(1.1)$ & 32 & 0 \\
\hline & HPV51 & $73(2.5)$ & 73 & 0 \\
\hline & HPV52 & $56(1.9)$ & 55 & 1 \\
\hline & HPV53 & $101(3.4)$ & 100 & 1 \\
\hline & HPV56 & $36(1.2)$ & 35 & 1 \\
\hline & HPV58 & $53(1.8)$ & 51 & 2 \\
\hline & HPV59 & $25(0.8)$ & 24 & 1 \\
\hline & HPV66 & $61(2.1)$ & 61 & 0 \\
\hline & HPV68 & $23(0.8)$ & 22 & 1 \\
\hline \multirow{6}{*}{ Low-risk } & HPV6 & $329(11.1)$ & 313 & 16 \\
\hline & HPV11 & $81(2.7)$ & 78 & 3 \\
\hline & HPV42 & $10(0.3)$ & 9 & 1 \\
\hline & HPV43 & $5(0.2)$ & 5 & 0 \\
\hline & HPV44 & $11(0.4)$ & 11 & 0 \\
\hline & HPV81 & $39(1.3)$ & 38 & 1 \\
\hline
\end{tabular}


Table 3. The Profile of HPV Frequency and HPV Genotypes Distribution in Iranian Women

\begin{tabular}{|c|c|c|c|c|c|c|c|c|}
\hline \multirow[b]{2}{*}{ Place } & \multicolumn{3}{|c|}{ Samples data } & \multicolumn{3}{|c|}{ Prevalence (\%) } & \multirow{2}{*}{ High frequent type } & \multirow{2}{*}{ Ref. } \\
\hline & Time & Size & Subjects & HPV & HPV16 & HPV18 & & \\
\hline Shiraz & $1999-2000$ & 101 & Cervical carcinoma & 87.1 & 26.7 & 0 & HPV16 & [42] \\
\hline Tehran & 2005-2006 & 127 & Outpatients & 31.5 & 23.6 & 3.2 & HPV16>HPV18 & [43] \\
\hline Tehran & 2005-2006 & 7 & Cervical carcinoma & 100 & 85 & 28 & HPV16>HPV18 & [43] \\
\hline Bushehr & 2008-2009 & 200 & Healthy women & 5.5 & 3.5 & 1.5 & HPV16>HPV18>HPV53 & [34] \\
\hline Zabol & $2006-2007$ & 265 & Gynecology outpatient clinic & 32.5 & 9 & 9.4 & HPV18> HPV16 & [40] \\
\hline Shiraz & 2010 & 402 & Healthy women & 5.5 & 2 & 0 & HPV16 & [44] \\
\hline Tehran & 2010-2012 & 851 & Gynecology outpatient clinic & 31.1 & 7.3 & 2.8 & HPV16> HPV 18 & [41] \\
\hline Gorgan, & 2011 & 378 & Gynecology outpatient clinic & 20.1 & 5.8 & 4 & HPV16> HPV18 & [45] \\
\hline Tehran & 2011 & 825 & Healthy women & 6.6 & 1.8 & 0.3 & HP16>HPV45>HPV31 & [35] \\
\hline Tehran & 2011 & 45 & Cervical carcinoma & 35.3 & 8.8 & 0 & HPV16=HPV58 & [35] \\
\hline Tehran & 2011-2013 & 436 & Cervical lesions & 45.4 & 32.8 & 5 & HPV16> HPV53>HPV31=HPV39 & [46] \\
\hline
\end{tabular}

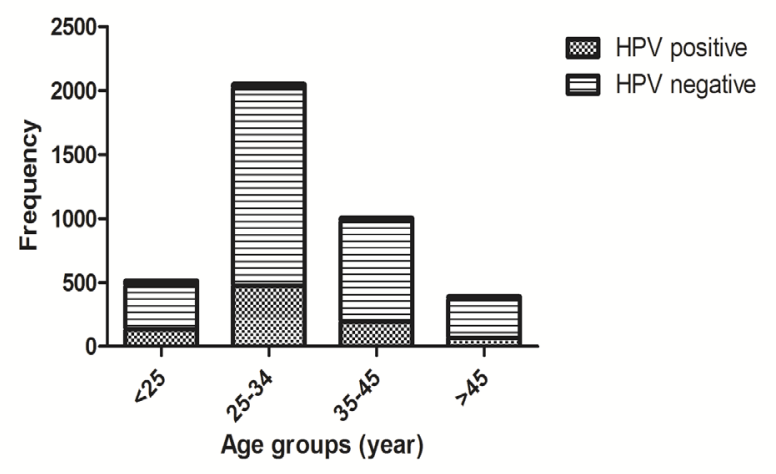

Figure 1. Age Specific Prevalence of HPV in Liquid Based Samples of Iranian Women

and abnormal groups.

\section{Discussion}

Some HPV types are well-accepted as the main etiological factors of cervical carcinoma which have been detected in almost all cervical cancer cases [29]. Besides cervical cancer, HPV virus has also been recognized as a definite cause of penis, vulva, vagina, anus, and oropharynx cancers [30-31]. In this context, HPV genomes integration into human genomes is a critical point of carcinogenesis process that leads to the viral proteins expression and induction of normal cell transformation [32].

HPV infection was associated with $4.8 \%$ of estimated 12.7 million new cancers in 2008 worldwide which considerably had higher incidence and mortality rates in developing countries compared to developed ones [33].

Bearing in mind the importance of HPV in cancer etiology, awareness of HPV prevalence as well as frequency of high-risk genotypes could help improve healthcare system management in order to prevent the related cancers. Thus, we retrospectively analyzed HPV prevalence in 2969 liquid-based samples (swap and genital wart) of women from different provinces of Iran that is the largest sample till now to the best of our knowledge.
In this regard, our results signified that HPV virus was detected in $29.3 \%$ of women referred by physicians to Noor pathobiology lab. In other studies, HPV prevalence was reported in 20.1-32.5\% of Iranian women attended to gynecology clinics (Table 3). Our finding is almost compatible with these reports; however, it is noteworthy to be noticed that this rate is higher than normal population of Iranian women with HPV infections [34-35]. In a meta-analysis study of HPV prevalence in healthy women from different provinces of Iran, it was reported that total prevalence of HPV was $9.4 \%$ with $2.0 \%, 1.7 \%$, and $5.3 \%$ accounted for HPV 16 , HPV 18 , and other genotypes, respectively [27-36]. In an elegant study, the prevalence of HPV infection was globally calculated between $11 \%-12 \%$ among women without cervical abnormalities. The highest rates of HPV infection were reported for sub-Saharan Africa, Eastern Europe, Latin America, and South-Eastern Asia with incidence of $24.0 \%$, $21.4 \%, 16.1 \%$, and $14.0 \%$, respectively [30-36-37].

Analysis of HPV positive cases showed that high risk and low-risk HPV subtypes were categorized into $67.2 \%$ and $52.0 \%$ of samples, respectively. Totally, HPV16, HPV18, and other HPV genotypes were found in $6.0 \%, 2.1 \%$, and $25.9 \%$ of samples, respectively (Table 2). Among high-risk HPV types, HPV16 (6.0\%) was confirmed as predominate type followed by HPV53 (3.4\%) and HPV39 (2.6\%) (Table 2). In comparison with our findings, Pouryasin (2014) showed that HPV53 followed by HPV16, HPV66, HPV18, HPV52, and HPV39 as the most prevalent genotypes in liquid-based samples of women from Tehran [38]. In other study in Tehran, prevalent HPV genotypes belonged to HPV16 (1.8\%), HPV45 (0.8\%), and HPV31 (0.6\%) in normal population [35]. In a Meta-analysis, HPV 16 (54\%), HPV18 (14\%), and HPV31 $(6 \%)$ were shown as the most frequently detected types in Iranian patients with cervical cancer [39]. Comparing to the worldwide HPV distribution, HPV16 (3.2\%), HPV18 (1.4\%), HPV52 (0.9\%), HPV31 $(0.8 \%)$, and HPV58 $(0.7 \%)$ were the most widespread HPV types $[30,36]$. These findings demonstrated that the pattern of HPV subtypes distribution could be varied among different regions. 
Regarding HPV prevalence in age groups, compatible with our finding, Shahramian (2011) reported that the most $\mathrm{HPV}$ infection was detected in married women at $15-25$ years age group at Zaboul located at South -Eastern of Iran. They also observed a decreasing trend of HPV frequency in older subgroups of $26-35,36-45$, and $>45$ years [40]. Shafaghi (2013) indicated that 19 HPV types were detected in $31.1 \%$ of Iranian women attended gynecology outpatient clinic [41]. Their finding demonstrated the highest prevalence of HPV in young women in 18-25 years old subgroups which encountered a decrease in the HPV frequency in older subgroups [41]. Furthermore, they showed that the prevalence of HPV16 and HPV18 were $7.3 \%$ and $2.8 \%$, respectively [41]. Their findings have overall compatibility with ours. In addition, the HPV distribution in different age groups has diverse patterns in the world with a higher prevalence in the younger ages ( $<25$ years) and a return at older ages ( $\geq 45$ years) in the Africa and Americas [36].

In conclusion, this study generated valid data regarding HPV prevalence in Iranian women from 24 different provinces. The large sample size and appling a standard and approved commercial HPV diagnostic kit were two important encouraging points of our study which let compare our findings with others. In overall, it can be concluded that HPV infection is currently at a considerable high level in Iran. Looking at the high risk and oncogenic HPV subtypes frequency in studied samples especially in younger age groups, a concern could be notified about HPV relevant cancers which can be prevented by commercial and approved HPV vaccines.

\section{Conflict of Interest}

All authors declare no conflicts of interest.

\section{Acknowledgments}

This project has been financially supported by Noor pathobiology laboratory.

\section{References}

1. Panatto D, Amicizia D, Bragazzi NL, Rizzitelli E, Tramalloni D, Valle I, et al. Human Papillomavirus Vaccine: State of the Art and Future Perspectives. Advances in protein chemistry and structural biology. 2015;101:231-322.

2. de Villiers EM, Fauquet C, Broker TR, Bernard HU, zur Hausen H. Classification of papillomaviruses. Virology. 2004;324(1):17-27.

3. Szostek S, Klimek M, Zawilinska B, Rys J, Kope J, Daszkiewic E. Detection of human papillomavirus in cervical cell specimens by hybrid capture and PCR with different primers. Acta Biochim Pol. 2006;53(3):603-7.

4. Grahovac M, Racic I, Hadzisejdic I, Doric A, Grahovac B. Prevalence of human papillomavirus among Croatian women attending the regular gynecological visit. Coll Antropol. 2007;31 Suppl 2:73-7.

5. Nasseri S, Monavari SH, Keyvani H, Nikkhoo B, Vahabpour Roudsari R, Khazeni M. The prevalence of Human Papilloma Virus (HPV) infection in the oligospermia and azoospermic men. Med J Islam Repub Iran. 2015;29:272.
6. de Sanjose S, Diaz M, Castellsague X, Clifford G, Bruni L, Munoz N, et al. Worldwide prevalence and genotype distribution of cervical human papillomavirus DNA in women with normal cytology: a meta-analysis. Lancet Infect Dis. 2007;7(7):453-9.

7. Haedicke J, Iftner T. Human papillomaviruses, and cancer. Radiother Oncol. 2013;108(3):397-402.

8. Munoz N, Bosch FX, de Sanjose S, Herrero R, Castellsague $\mathrm{X}$, Shah KV, et al. Epidemiologic classification of human papillomavirus types associated with cervical cancer. N Engl J Med. 2003;348(6):518-27.

9. Jamshidi M, Shekari M, Nejatizadeh AA, Malekzadeh K, Baghershiroodi M, Davudian P, et al. The impact of human papillomavirus (HPV) types 6, 11 in women with genital warts. Arch Gynecol Obstet. 2012;286(5):1261-7.

10. Clifford GM, Gallus S, Herrero R, Munoz N, Snijders PJ, Vaccarella S, et al. Worldwide distribution of human papillomavirus types in cytologically normal women in the International Agency for Research on Cancer HPV prevalence surveys: a pooled analysis. Lancet. 2005;366(9490):991-8.

11. Cox JT. Epidemiology and natural history of HPV. J Fam Pract. 2006; Suppl:3-9.

12. von Krogh G. Management of anogenital warts (condylomata acuminata). Eur J Dermatol. 2001;11(6):598-603; quiz 4.

13. Foresta C, Garolla A, Zuccarello D, Pizzol D, Moretti A, Barzon L, et al. Human papillomavirus found in sperm head of young adult males affects the progressive motility. Fertil Steril. 2010;93(3):802-6.

14. Robinson D, Coupland V, Moller H. An analysis of temporal and generational trends in the incidence of anal and other HPV-related cancers in Southeast England. Br J Cancer. 2009;100(3):527-31.

15. Hamkar R, Azad TM, Mahmoodi M, Seyedirashti S, Severini A, Nategh R. Prevalence of human papillomavirus in Mazandaran Province, Islamic Republic of Iran. East Mediterr Health J. 2002;8(6):805-11.

16. Hamkar R M-AT, Mahmoodi M, Seyedirashti S, Severini A, Nategh R Prevalence of various types of HPV among cervical cancer and normal biopsy in the North of Iran. Iranian Journal of Infectious Diseases and Tropical Medicine. 2004;8(22):9-16.

17. Walboomers JM, de Roda Husman AM, Snijders PJ, Stel HV, Risse EK, Helmerhorst TJ, et al. Human papillomavirus in false negative archival cervical smears: implications for screening for cervical cancer. J Clin Pathol. 1995;48(8):72832.

18. Vinodhini K, Shanmughapriya S, Das BC, Natarajaseenivasan $\mathrm{K}$. Prevalence and risk factors of HPV infection among women from various provinces of the world. Arch Gynecol Obstet. 2012;285(3):771-7.

19. Jahdi F, Khademi K, Merghati Khoei E, Haghani H, Yazdanpanahi Z. Evaluation of the Individual and Medical Factors Associated With Genital Human Papillomavirus in Iranian Women. Scimetr 2014;2(4):e17495

20. Mbulawa ZZ, Coetzee D, Williamson AL. Human papillomavirus prevalence in South African women and men according to age and human immunodeficiency virus status. BMC Infect Dis. 2015;15:459.

21. Tay SK, Tay YK. The prevalence and significance of high-risk human papillomavirus DNA test in southern Malaysia and Singapore. Aust N Z J Obstet Gynecol. 2009;49(3):323-7.

22. Monsonego J, Zerat L, Syrjanen K, Zerat JC, Smith JS, Halfon P. Prevalence of type-specific human papillomavirus infection among women in France: Implications for screening, vaccination, and a future generation of multivalent 
HPV vaccines. Vaccine. 2012;30(35):5215-21.

23. Shipitsyna E, Zolotoverkhaya E, Kuevda D, Nasonova V, Romanyuk T, Khachaturyan A, et al. Prevalence of highrisk human papillomavirus types and cervical squamous intraepithelial lesions in women over 30 years of age in St. Petersburg, Russia. Cancer Epidemiol. 2011;35(2):160-4.

24. Zeng XX, Yan LX, Huang XX, He CH, Liu WG, Yuan WQ, et al. Prevalence and genotype distribution of human papillomavirus among Hakka women in China. Ann Transl Med. 2016;4(15):276.

25. Balanda M, Quiero A, Vergara N, Espinoza G, Martin HS, Rojas G, et al. Prevalence of human papillomavirus infection among women presenting for cervical cancer screening in Chile, 2014-2015. Med Microbiol Immunol. 2016;205(6):585-94.

26. Santos Filho MV, Gurgel AP, Lobo CD, Freitas AC, SilvaNeto JC, Silva LA. Prevalence of human papillomavirus (HPV), distribution of HPV types, and risk factors for infection in HPV-positive women. Genet Mol Res. 2016;15(2).

27. Malary M, Moosazadeh M, Hamzehgardeshi Z, Afshari M, Moghaddasifar I, Afsharimoghaddam A. The Prevalence of Cervical Human Papillomavirus Infection and the Most At-risk Genotypes Among Iranian Healthy Women: A Systematic Review and Meta-analysis. Int J Prev Med. 2016;7:70.

28. WHO/ICO Information Centre on HPV and Cervical Cancer (HPV Information Centre). Human Papillomavirus and Related Cancers in Iran. Summary Report 2010. [Available from http://screening.iarc.fr/doc/ Human\%20 Papillomavirus\%20and\%20Related\%20 Cancers.pdf.

29. Burk RD, Chen Z, Van Doorslaer K. Human papillomaviruses: genetic basis of carcinogenicity. Public Health Genomics. 2009;12(5-6):281-90.

30. Forman D, de Martel C, Lacey CJ, Soerjomataram I, Lortet-Tieulent J, Bruni L, et al. Global burden of human papillomavirus and related diseases. Vaccine. 2012;30 Suppl 5: F12-23.

31. Reiter PL, Pendergraft WF, 3rd, Brewer NT. Meta-analysis of human papillomavirus infection concordance. Cancer Epidemiol Biomarkers Prev. 2010;19(11):2916-31.

32. Liu CY, Li F, Zeng Y, Tang MZ, Huang Y, Li JT, et al. Infection and integration of high-risk human papillomavirus in HPV-associated cancer cells. Med Oncol. 2015;32(4):109.

33. Bosch FX, Broker TR, Forman D, Moscicki AB, Gillison ML, Doorbar J, et al. Comprehensive control of human papillomavirus infections and related diseases. Vaccine. 2013;31 Suppl 6: G1-31.

34. Zandi K, Eghbali SS, Hamkar R, Ahmadi S, Ramedani E, Deilami I, et al. Prevalence of various human papillomavirus (HPV) genotypes among women who subjected to routine Pap smear test in Bushehr city (south west of Iran) 20082009. Virol J. 2010;7:65.

35. Khodakarami N, Clifford GM, Yavari P, Farzaneh F, Salehpour S, Broutet N, et al. Human papillomavirus infection in women with and without cervical cancer in Tehran, Iran. International journal of cancer. 2011;131(2): E156-61.

36. Bruni L, Diaz M, Castellsague X, Ferrer E, Bosch FX, de Sanjose S. Cervical human papillomavirus prevalence in 5 continents: a meta-analysis of 1 million women with normal cytological findings. J Infect Dis. 2010;202(12):1789-99.

37. WHO. Human papillomavirus vaccines: WHO position paper, October. 2014 NO. 43,

38. Pouryasin M, Sharafi H, Mousavi AS, Khodadad S, Marjani M, Jamshidi F, et al. Distribution of Human Papillomavirus
Genotypes in Liquid-based Samples; Abundance of HPV-53 in Tehran, Iran. Iran J Public Health. 2014;43(8):1159-60.

39. Khorasanizadeh F, Hassanloo J, Khaksar N, Mohammad Taheri S, Marzaban M, B HR, et al. Epidemiology of cervical cancer and human papillomavirus infection among Iranian women - analyses of national data and systematic review of the literature. Gynecol Oncol. 2010;128(2):277-81.

40. Shahramian I, Heidari Z, Mahmoudzadeh-Sagheb H, Moradi A, Forghani F. Prevalence of HPV Infection and High-Risk HPV Genotypes $(16,18)$, among Monogamous and Polygamous Women, In Zabol, Iran. Iran J Public Health. 2011;40(3):113-21.

41. Shafaghi B JA, Yousefzadeh B, Ameri A, Moghadam S, Mostafavi M. Human Papilloma Virus Prevalence and Types among Iranian Women Attending Regular Gynecological visits. Reports of Radiotherapy and Oncology. 2013;1(2):7.

42. Farjadian S, Asadi E, Doroudchi M, Dehaghani AS, Tabei SZ, Kumar VP, et al. High-isk HPV types in southern Iranian patients with cervical cancer. Pathol Oncol Res. 2003;9(2):121-5.

43. Ghaffari SR, Sabokbar T, Mollahajian H, Dastan J, Ramezanzadeh F, Ensani F, et al. Prevalence of human papillomavirus genotypes in women with normal and abnormal cervical cytology in Iran. Asian Pac J Cancer Prev. 2006;7(4):529-32.

44. Safaei A, Khanlari M, Momtahen M, Monabati A, Robati M, Amooei S, et al. Prevalence of high-risk human papillomavirus types 16 and 18 in healthy women with cytologically negative pap smear in Iran. Indian J Pathol Microbiol. 2010;53(4):681-5.

45. Moradi A, Bakhshandeh Nosrat S, Besharat S. Molecular Epidemiology of High-Risk Types of Human Papillomaviruses $(16,18)$ in Pap-Smear, the North East of Iran. Iran J Cancer Prev. 2011;4(3):135-40.

46. Salehi-Vaziri M, Sadeghi F, Hashemi FS, Haeri H, BokharaeiSalim F, Monavari SH, et al. Distribution of Human Papillomavirus Genotypes in Iranian Women According to the Severity of the Cervical Lesion. Iran Red Crescent Med J. 2016;18(4):e24458.

This work is licensed under a Creative Commons AttributionNon Commercial 4.0 International License. 